\title{
人在回路的在线手写数学公式识别方法
}

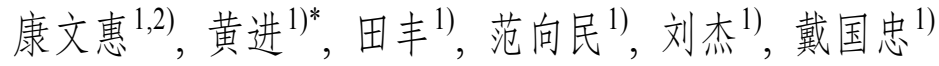 \\ 1) (中国科学院软件研究所人机交互北京市重点实验室 北京 100190) \\ 2) (中国科学院大学计算机科学与技术学院 北京 100049) \\ (huangjing@iscas.ac.cn)
}

\begin{abstract}
摘 要: 在线手写数学公式识别面临书写字符的不确定性、数学公式结构的复杂性, 以及公式书写风格因人而异等 问题, 特别是在公式书写中出现偶然性错误和包含复杂结构的情况下, 现有的仅依赖机器的识别算法的识别准确率 较低. 为了解决这一问题，提出了人在回路的手写公式识别方法，该方法主要在结构分析阶段引人了人的参与，借助 人对结构中歧义笔画的修改和结构补笔操作，完善和界定结构笔画和结构内笔画信息. 为了评估该方法的有效性， 将其与不含用户参与信息的一个基线识别方法在结构识别率和表达式识别率方面进行了对比分析. 结果表明, 该方 法能够有效地促进用户参与到手写识别过程，同时，针对实验收集的手写数学公式数据，引人用户参与的方法能够 有效地提高手写数学公式的结构和表达式识别率，分别提高了 $9.26 \%$ 和 $13.99 \%$.
\end{abstract}

关键词: 人在回路; 手写数学公式; 草图识别; 人机交互; 用户反馈

中图法分类号: TP391. $41 \quad$ DOI: $10.3724 /$ SP.J.1089.2021.18796

\section{Human-in-the-Loop Based Online Handwriting Mathematical Expressions Recognition}

\author{
Kang Wenhui ${ }^{1,2)}$, Huang Jin ${ }^{1 *}$, Tian Feng ${ }^{1)}$, Fan Xiangmin ${ }^{1)}$, Liu Jie ${ }^{1)}$, and Dai Guozhong ${ }^{1)}$ \\ 1) (Beijing Key Laboratory of Human-Computer Interaction, Institute of Software, Chinese Academy of Sciences, Beijing 100190) \\ 2) (School of Computer Science and Technology, University of Chinese Academy of Sciences, Beijing 100049)
}

\begin{abstract}
Online handwritten mathematical expression recognition method faces some challenges, such as the uncertainty of writing characters, the complexity of mathematical expression structure and the various written styles of expression. Existing no-human-involved recognition algorithms have low recognition accuracy, especially when accidental errors and complex structures occur in mathematical expression writing. A human-inthe-loop based online handwritten mathematical expression recognition method is proposed, which mainly introduces human participation in the structural analysis stage to define and perfect the structural strokes or strokes within the mathematical structure, by means of human modification for the ambiguous strokes in the structure and operation of structural stroke makeup. To evaluate the effectiveness of the proposed method, the method is compared with a no-human-involved recognition algorithm in rate of structural analysis and expression recognition rate. The results show that proposed method can effectively promote users to participate in the process of recognition, and that incorporating user in the recognition processes can improve the accuracy of recognition of structure
\end{abstract}

收稿日期：2020-11-23; 修回日期：2021-05-01. 基金项目：国家自然科学基金(61802379); 中国科学院前沿基础研究重点项目 (QYZDY-SSW-JSC041); 中国科学院青年创新促进会项目(2020113). 康文惠(1993一), 男, 博士研究生, 主要研究方向为人机混合智 能、草图理解; 黄进(1985-), 男, 博士, 助理研究员, CCF 会员, 论文通讯作者, 主要研究方向为人机交互、图形图像处理; 田丰 (1976-), 男, 博士, 研究员, 博士生导师, CCF 会员, 主要研究方向为人机交互、虚拟现实; 范向民(1989一), 男, 博士, 副研究员, CCF 会员, 主要研究方向为人机交互、智能用户界面; 刘杰(1981一), 男, 博士, 高级工程师, CCF 会员, 主要研究方向为人机交互、 计算机图形学; 戴国忠(1944-), 男, 学士, 研究员, 博士生导师, CCF 会员, 主要研究方向为人机交互、计算机图形学. 
$(9.26 \%)$ and expression (13.99\%) in handwritten mathematical expression.

Key words: human-in-the-loop; handwritten mathematics; sketch recognition; human-computer interaction; user feedback

自 20 世纪 50 年代以来, 手写数学识别作为物 理学、几何定理证明和代数智能教学系统等众多基 于笔的软件系统的关键组成部分, 一直是草图识 别方面比较活跃的研究领域 ${ }^{[1]}$. 近年来, 随着触摸 屏和笔式设备的普及, 手写数学表达式识别在教 育、办公自动化和会议系统等许多方面具有广泛的 潜在应用, 因此逐年稳步吸引着越来越多的社区 关注，也促进了具有手写识别和手绘交互界面的 软件研发 ${ }^{[2]}$. 但是, 用户在使用这些系统时对手写 自由度和识别精度有很高的要求, 对手写数学识 别的系统研究表明 ${ }^{[3]}$, 手写数学识别需要 $91.00 \%$ $97.00 \%$ 的识别率才能被接受. 但是根据第 15 届手 写数学表达识别与排版公式检测竞赛 ${ }^{[4]}$ 和第 17 届 国际笔迹识别前沿会议关于手写数学表达式离线 识别国际竞赛 ${ }^{[5]}$ 的结果来看, 无论是在线识别方法 还是离线识别方法, 其表达式识别率均低于用户 可接受的最低识别率, 难以满足用户的基本要求.

在实际情况下, 离线手写数学表达式识别可 能会遇到模糊、噪声、缺少笔触和背景复杂等问题, 且不能满足用户实时识别和对手写数学公式进行 交互式修改的需求. 因此, 研究内容聚焦于在线手 写识别的方法, 这种方法可以减少手写体模糊带 来的困难，同时可以利用丰富的动态(时间和空间) 信息. 在线手写数学表达式识别包含字符识别和 结构分析 ${ }^{[6]} 2$ 个主要处理过程. 但用户书写字符顺 序不一致、书写字符风格迥异、数学公式结构信息 复杂和全局信息缺乏等问题, 为在线识别数学公 式带来了挑战. 不管是机器学习方法 ${ }^{[7]}$, 还是基于 “符号分割-符号识别-结构分析”的传统算法 ${ }^{[8]}$, 都 很难解决由于用户书写内容不确定、笔画重叠或交 叉以及公式中相似字符等问题带来的识别难题.

书写过程中的字符识别错误或公式结构识别 错误都会打断书写过程, 干扰用户的思考, 降低用 户对识别系统的满意度. 为了解决识别错误的问 题, 相关研究除了提高算法识别率外, 还有针对约 束和规范用户书写方式的诸多研究, 如基于笔画 顺序规范化的在线手写数学表达式识别方法 ${ }^{[9]}$ 、可 视化字符或字符块的数学框 ${ }^{[10]}$ 等. 但是通过引人 人的参与解决手写数学公式识别问题的研究较少, 且大多数研究停留在通过删除笔画和重写笔画 ${ }^{[8]}$ 等方式解决书写内容错误或书写字迹过于潦草的
问题, 并没有在数学公式识别过程中引人人干预. 通过对用户的书写习惯分析发现, 即使预先 提供要书写的公式, 用户也不会在事先分配足够 的空间书写出完整的表达式 ${ }^{[10]}$, 如图 1 所示, 分式 表达式中分数线短而引起识别错误. 更糟糕的是, 当这些问题确实发生时, 删除部分笔画或重写可 能不足以纠正所有的错误. 同时, 根据最新字符识 别研究, 字符识别准确率能够达到 $91.50 \%{ }^{[11]}$, 但 是数学表达式识别准确率仍然很低, 是因为数学 表达式识别不仅需要字符识别, 还需要识别字符 之间的空间结构, 而公式结构的识别率低下一直 是制约数学表达式识别的重要因素. 因此, 本文提 出了人在回路 ${ }^{[12]}$ 的手写数学公式识别方法, 人在 回路的方法主要是借助人的因素, 在合适的时机 引人人的参与, 与机器共同完成任务. 在手写数学 公式识别中, 除了对输人笔画的删除外, 在结构分 析阶段也引人了人的参与, 通过借助人对结构中 歧义笔画的修改和结构补笔操作, 完成对结构笔 画的完善和对结构内笔画信息的界定，进而提高 结构和表达式识别率. 随后与用户不参与识别过 程的算法进行了评估和比较.

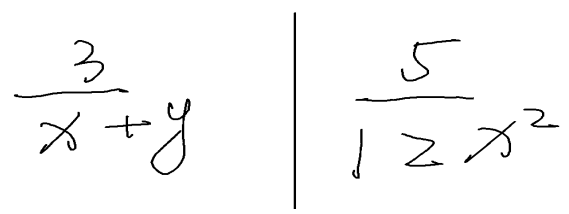

图 1 分式表达式中分数线短问题

本文的主要贡献如下.

(1) 提出了一种人在回路的手写数学公式识 别方法, 该方法充分利用了人的反馈信息.

(2) 验证了手写数学公式识别过程中引人人 的参与协助识别的可行性.

(3) 展示了通过引人人的参与解决手写公式 识别的能力.

\section{1 相关研究}

\section{1 手写数学公式识别}

手写数学公式的识别多年来一直是一个活跃 的研究领域, 识别过程可以看做将手写笔画的二 
维语言翻译成计算机可以使用的序列形式, 如 LATEX 或 MathML 等数学描述语言. 经典识别方 法是基于结构化方法的，识别任务被视为解决符 号分割、符号识别和结构解析 3 个任务, 结构解析 是在符号分割和分类结构的基础上进行的. 对于字 符分割任务，常用的方法有基于生成树的方法、贝 叶斯模型和基于图的方法等; 符号识别算法包括最 近邻算法、隐马尔可夫模型、支持向量机和递归神 经网络等; 对于结构解析, 提出的特征包括符号边 界框、相对大小和位置，以及极性直方图等 ${ }^{[13-14]}$. 但 是这种方法上一级的错误会对下一级任务产生严重 的影响, 错误会逐级传播下去, 如来自符号分割和 识别的错误会传播到结构分析中. 同时, 数学表达 式识别中结构分析一直是最具挑战性的问题 ${ }^{[15]}$.

随着深度学习在图像和自然语言处理中取得 突破性进展，一些基于端到端的深度学习方法也 被应用于手写数学表达式识别, 如基于残差双向 递归神经网络的 Seq2Seq 模型 ${ }^{[6]}$ 、基于树的双向长 短时记忆网络 ${ }^{[13]}$ 、笔画限制注意力网络 ${ }^{[16]}$ 和模糊 神经网络 ${ }^{[17]}$ 等方法. 这些方法将字符识别和结构 分析融为一体，采用端到端编码解码的方式识别 手写数学公式, 但是它们均严重依赖数据集的数 量和质量, 且难以对模型进行解释. 在识别率方 面, 基于深度学习的在线手写公式识别方法在数 学表达式上表现不佳, 其识别正确率基本保持在 $30.00 \% \sim 80.00 \%{ }^{[6-7,16]}$.

总体而言，不管是采用基于结构化的经典方 法还是基于端到端的深度学习方法, 都难以满足 用户对手写数学公式系统的需求. 鉴于此, 研究人 员另辟蹊径，考虑从笔式交互用户界面的角度提 高手写数学公式的识别率 ${ }^{[18]}$. 基于笔的用户界面 Math Boxes ${ }^{[10]}$, 在检测到符号(包括上标、下标和 分数)之间的相关空间关系时, 将自动生成子表达 式周围的可见边界框, 且数学框中包含的子表达 式可以通过直接向其给定边界添加新项来扩展; 基于流体笔的数学输人和编辑系统 ${ }^{[19]}$, 支持删除 笔画、拖动笔画和计算功能. 同时也有学者在逐步 探索以笔为中心的智能用户界面对数学表达式操 作的新要求 ${ }^{[2]}$, 主要探索了连写、变量使用和编辑 函数有关的几个方面. 但是, 像 MathBoxes 这类显 式交互的规范方式，必然引人了一个高负荷的认 知步骤——用户必须从思考自己手头问题转向检 查输人表达式的空间关系

\section{2 人在回路的方法}

人在回路的方法描绘了一个机器和用户为了
提高系统执行能力(表现)的交互场景, 其基本思路 是机器根据已有信息(通常具有不确定性、多维等 特点)预测或分类出一个初始结果, 如果结果错误, 则人通过自我认知和推理能力纠正错误结果并反 馈于机器, 同时机器结合用户反馈信息来改善预 测结果, 从而减少人的负担 ${ }^{[20]}$. 该方法主要解决 过分依赖机器自动化而忽略人的因素 (自动化偏 见)的问题，帮助用户防范算法错误造成的有害影 响, 特别是在一些关乎生命和安全的场景中 ${ }^{[21]}$. 尽管在智能机器的帮助下人做出的决策结果并不 一定比人单独做出的决策好 ${ }^{[13]}$, 但是已有研究表 明 ${ }^{[22-24]}$, 人类能够确定机器建议错误的某些情况, 并且能够在这种情况下适当地取代机器的结果.

为了解决机器智能无法满足实际问题出现的 状况, 如机器模型在面对没有遇见的新产品或新 数据时, 将以非常低的置信度进行预测结果等问 题, 研究人员探索了人机互补的方法, 并根据实际 问题设计了结合两者优点的方法. 根据最新研究 发现，人在回路的方法常应用到儿童虐待热线篮 查辅助决策 ${ }^{[12]}$ 、病例分类和医疗诊断 ${ }^{[25]}$ 、智能答 疑系统 ${ }^{[26]}$ 、飞行模拟器 ${ }^{[27]}$ 、服务台聊天机器人 ${ }^{[28]}$ 和自动驾驶汽车 ${ }^{[29]}$ 等方面.

在数学公式识别方面, 现有的技术更多关注 给定的工作流(字符分割、字符识别和结构解析)的 规范和加速 ${ }^{[6-9]}$ ，并没有考虑“人在回路”方面的迭 代, 忽略了用户指导和辅助机器在公式识别方面 的探索. 特别是在面对用户输人信息模糊和不确 定的情况下，现有技术无法满足用户的需求 ${ }^{[3]}$. 因 此，对于仅依赖识别算法不能满足用户对手写公 式识别的要求, 而基于笔式手写数学公式识别界 面的研究更多关注如何规范和约束用户手写方式, 以及现有的交互技术主要用于删除、重写笔画等问 题, 本文结合手写数学公式识别的 3 个阶段和人在 回路的基本思路，提出了人在回路的手写公式识 别方法. 其不仅包含对输人笔画删除重写等交互 式操作, 而且在结构分析阶段引人人的主动参与, 使用户借助自然且符合人书写习惯的交互方式, 帮助识别算法减少数学空间结构的不确定, 特别 是数学分式和根式结构的不确定.

\section{2 基于人在回路的识别方法}

\section{1 基础方法}

本文采用 Seshat ${ }^{[30]}$ 作为基础方法，用于研究 人在回路中的手写数学公式识别. Seshat 是用于识 
别手写数学表达式的开源算法 ${ }^{1}$, 它在 2014 年 ICFHR 竞赛中被评为竞赛数据集上训练的最佳在 线手写公式识别算法. 之所以采用 Seshat 算法, 是 因为它是传统 3 阶段方法应用到在线手写数学公式 识别中识别率最高的算法. 随着机器学习的发展, 研究人员尝试将深度学习方法应用到手写数学公式 识别中 ${ }^{[4-5,13,16]}$, 并取得了较高的公式识别率. 但是 目前深度学习方法很难引人人的参与, 并结合人 的反馈信息调整模型.

Seshat 算法采用“符号分割-符号识别-结构解 析”的传统框架完成手写数学表达式识别, 其目的 在于识别一组笔画序列为 $O=o_{1} O_{2} \cdots o_{n}$ 所包含的字 符 $S=s_{1} S_{2} \cdots s_{n}$, 并根据字符间的空间关系和数学 规则构造解析树, 识别结果为解析树中最大可能 概率的解析树 $t$, 其形式化表达为

$$
\hat{t}=\underset{t \in T}{\arg \max } \max _{s \in E} p(S \mid O) p(t \mid S) .
$$

其中, $T$ 为所有可能的解析树集合; $E$ 为所有的数学 符号.

\section{1 .1 符号分割}

数学表达式的许多符号是由多个笔画组成的, 且这些笔画在空间上非常接近. 例如, “六”由 3 个笔 画组成. Seshat 算法采用基于空间和几何信息的方 法完成符号分割, 具体过程如下.

Step1. 查找接近笔画组. 采用欧几里得度量计算 2 个笔画最近点之间的距离 $d$, 并设定最大距离阈值 $L$ 判 断笔画是否接近.

Step2. 判断是否为可见笔画. 如果 2 个笔画的最近 点之间的直线没有穿过任何其他笔画, 则认为这 2 个笔 画是可见的.

Step3. 判断笔画分割假设是否被接受. 以笔画为 节点, 以可见且接近的笔画之间的连线为边构造无向图 $G$. 如果笔画分割假设 $b=\left\{b_{i}\left|b_{i} \in O,\right| b_{i} \mid \leqslant L_{\text {max }}\right\}$ 是 $G$ 的 一个连通子图, 则该假设是可接受的. 其中, $L_{\text {max }}$ 为可 接受假设最大笔画数.

Step4. 计算不同笔画分割假设形成数学符号的概 率 $p\left(b_{i}\right)=p_{\mathrm{GMM}}\left(c=1 \mid g\left(b_{i}\right)\right)$. 其中, $c=1$ 表示可接受假 设 $b_{i}$ 的笔画能够形成数学符号. GMM 为高斯混合模型 (Gaussian mixture model).

\subsection{2 符号识别}

符号识别是正确识别数学符号的关键. Seshat 算法采用双向长短时记忆循环神经网络(bi-directional long short-term memory recurrent neural networks, BLSTM-RNN)进行数学符号分类. 其模型 训练过程利用在线特征和离线特征分别训练
BLSTM-RNN 模型, 并通过线性插值和权重参数 组合这 2 个分类器. 其中, 在线特征包括标准化坐 标、标准化一阶导、标准化二阶导和曲率; 离线特 征是从一个分割假设集生成的高为 $H$ 且宽为 $5 H$ 的图像中提取的, 包括列中黑色像素数、列的重心、 列的二阶矩等 ${ }^{[31]}$. 符号识别模型的最终概率为

$$
p\left(s_{i} \mid b_{i}\right)=\alpha \cdot p_{\text {on }}\left(s_{i} \mid b_{i}\right)+(1-\alpha) \cdot p_{\text {off }}\left(s_{i} \mid b_{i}\right) .
$$

其中, $s_{i}$ 为识别的字符; $p_{\text {on }}\left(s_{i} \mid b_{i}\right)$ 为在线模型的 识别概率; $p_{\text {off }}\left(s_{i} \mid b_{i}\right)$ 为离线模型的识别概率.

\subsection{3 结构解析}

对于用户输人的一系列笔画, 数学表达式识 别器的输出通常是一系列包含符号间关系结构的 数学字符, 但是解析数学公式字符间的空间关系 是一项具有挑战的任务. Seshat 算法将数学表达式 识别问题视为如何获得给定笔画序列最有可能的 解析树问题, 其解决方法为基于上下文无关文法 模型的二维扩展(probabilistic context free grammar, $P C F G)^{[15]}$ 构建生成模型. PCFG 由一个 4 元组 $(\aleph, \Sigma, S, P)$ 和对应概率组成. 其中, $\aleph, \Sigma, S, P$ 分别 为非终结符集合、终结符集合、语法开始符号和规 则. 结构解析步骤如下所示 ${ }^{[30]}$.

Step1. 定义空间关系模型. Seshat 算法使用 6 种空 间关系处理数学表达式, 包括左右、上下、上标、下标、 内部和根式.

Step2. 训练空间关系统计分类器. 该训练器依据 2 个非终结符编码区域的 9 个几何特征进行训练.

Step3. 计算空间关系概率. 通过分类器提供的概率 和基于分层聚类的惩罚函数 ${ }^{[32]}$ 完成空间关系概率的计算, 表示为 $p(r \mid B C)=p_{\mathrm{GMM}}(r \mid h(B, C)) \cdot f(B, C)$. 其中, $r$ 表 示为空间关系; $B$ 和 $C$ 为非终结符; $f(B, C)$ 为惩罚函 数, 用于首先合并最接近的笔画; $p(r \mid B C)$ 表示 $B$ 和 $C$ 的区域根据空间关系 $r$ 排列的概率.

Step4. 构建数学公式解析树. 基于 $\mathrm{CYK}^{[33]}$ 的 PCFG 计算最有可能的解析树, 解析楖概率计算为

$$
p(t, s)=\prod_{(A \rightarrow s, t)} p(\alpha \mid A) \prod_{(A \rightarrow B C, t)} \max _{r} p(B C \mid A) p(r \mid B C) .
$$

其中, $p(\alpha \mid A)$ 和 $p(B C \mid A)$ 表示语法规则的概率; $(A \rightarrow \alpha, t)$ 表示所有非终结符 $A$ 推导出终结符 $\alpha$ 的规则; $(A \rightarrow B C, t)$ 表示所有非终结符 $A$ 由 2 个非终结符 $B C$ 合 成的规则; 终结符 $\alpha$ 对应数学表达式中的数学符号 $s$.

\section{2 介入方式}

不管是采用基于结构化的经典方法还是基于 端到端的深度学习方法, 都难以满足用户对手写 数学公式系统的需求. 同时, 从用户界面角度设计 的方法更多关注如何规范用户输人, 或引人套索 
等手势实现交互. 但是从用户的角度出发, 用户 希望在书写过程中拥有较大的书写自由度, 而手 势类操作会提高用户的认知负荷(用户必须从思 考自己手头的问题转向考虑如何使用手势，且在 手势操作过程中需要检查操作结果是否符合他们 的意图).

因此，本文在识别过程中引人人的参与，并设 计了人在回路的介人方式, 如图 2 所示. 图 2 左侧 表示在算法识别过程中不包含人在回路方法的处 理过程, 用户只负责笔画输人和识别结果接收; 而 提出的人在回路方法(图 2 右侧)中，用户除了笔画 输人和结果接收外，还可以在识别算法的不同阶 段介人. 人参与过程在输人端包含 2 种方式: (1) 基于用户潜意识判定算法不能识别或者自己书写 有误情况下，删除部分笔画或重新书写已输人笔 画；(2) 根据识别结果对笔画进行修改, 特别是对 字符和结构的修改. 本文基于方式(2)的情况设计 了人参与识别介人方法，该方法提供交互式识别 反馈时，相信用户可以在输人过程中分推机器对 公式错误识别的检查. 本文方法有 3 个中心目标.

（1）用户在付出最小努力的情况下，灵活地参 与到公式识别过程中.

(2) 引人人的参与提高手写数学公式的识别率.

（3）在基础方法识别率低的情况下，满足用户 对手写公式识别的部分需求.

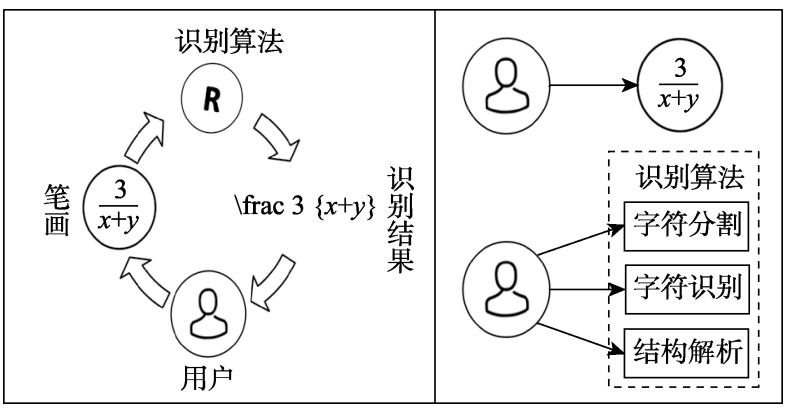

a. 不介人

b. 介人

图 2 识别过程用户不介人和介人的示意图

根据中心目标，本文定义了人在回路中涉及 的主要交互手段, 包括删除笔画、撤销上一笔、补 笔和清空, 其中删除、撤销和清空借助按钮完成. 具体如下

(1) 删除笔画，通过“删除”按钮触发删除操 作. 用户使用笔删除已存在的笔画或某一笔画的 部分点, 能够根据实际需求调整笔迹粗细. 主要解 决 2 个方面的问题：一是方便用户在书写错误或书
写不合心意时进行修改; 二是根据对已有笔画的 识别反馈，用户可以使用该方式删除引起识别错 误的笔画或修改某一笔画的局部区域，如因为连 笔引起的识别错误，通过删除连笔笔画的连接点 来更新识别结果.

（2）撤销上一笔. 除了解决初始书写时因前一 笔书写错误而需要完全删除该笔画问题外, 也可 以根据识别反馈信息来解决因前一笔的书写引起 结构识别错误而需要撤销的问题. 如图 3 所示, 上 下标结构公式“I"2”，因上标“2”的笔画在垂直方向 上过于靠近“I”，导致算法识别为“I2”而需要撤销 “2”的笔画.

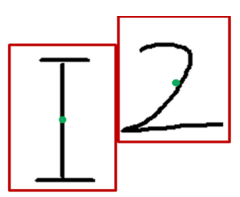

图 3 “I2”中上标“2”笔画在垂直方向上过于靠近“ I”

(3) 补笔操作. 用户在纸笔书写时, 通常会对 符号缺失笔画或不能完全包含分式根式结构信息 的分号根号, 采用再次补笔的方式准确地表达其 包含的信息和含义. 因此，本文仿照用户在纸笔书 写中的使用习惯设计了补笔操作, 以方便用户参 与到手写数学表达式识别中去. 补笔操作主要包 含符号补笔、分式补笔和根式补笔. 符号补笔主要 解决用户书写时由于个人书写习惯或书写速度过 快而引起的符号信息缺失问题，如由于书写速度 过快，“a”的笔画被识别为“u”，用户通过简单地在 符号“和中补笔完成符号“a”的正确识别.

分式表达式中分数线短而引起识别错误问题, 通过删除笔画或撤销上一笔很难解决识别错误, 且需要耗费更多的时间. 因此, 本文通过补笔的方 式引人人的参与, 用户通过延长分式符号完成补 笔操作. 类似于分式补笔, 针对根式符号不能完全 包含根式内容的问题，本文方法也可以采用补笔 操作引导用户参与到根式结构识别当中. 如图 4 所 示，用户的真实意图是书写 “ $\sqrt{x^{2}+2 x+6}$ ”, 由于 书写的根式符号太短而不能完全包含 “ +6 ” 的笔 画，被算法识别为 “ $\sqrt{x^{2}+2 x}+6$ ”. 因此，可以采用 本文提出的补笔操作进行补笔 $s_{15}$ 后, 算法结合上 下文信息可以判定补笔笔画为根式符号的一部分.

（4）清空. 主要解决无法通过局部修正已有笔 画来完成识别任务, 或局部修正时间远远超出重 写时间等导致的需要清除全部笔画等问题. 


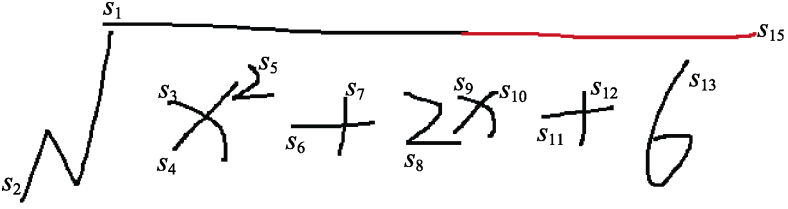

图 4 根式补笔示意图

\section{3 本文方法}

人在回路的手写数学表达式识别方法需要解 决 2 方面的问题：(1) 设计用户介人算法的方式, (2) 融合算法和用户交互信息. 本文采用删除、撤 销、补笔和清空等交互方式在识别算法中引人人的 参与, 特别是补笔操作.

对于算法和用户交互信息的融合，需要结合 手写数学表达式 $E$ 的上下文信息和用户意图完成 算法的调整和融合. 而如何融合算法和用户交互 信息是人在回路的识别方法中最具挑战的问题, 如根式补笔中补笔笔画是根式符号的补充笔画, 还是根式外符号的上横线. 针对如何结合已有识 别算法和用户参与信息，本文提出了基于上下文 信息的融合方法，基本流程图如图 5 所示.

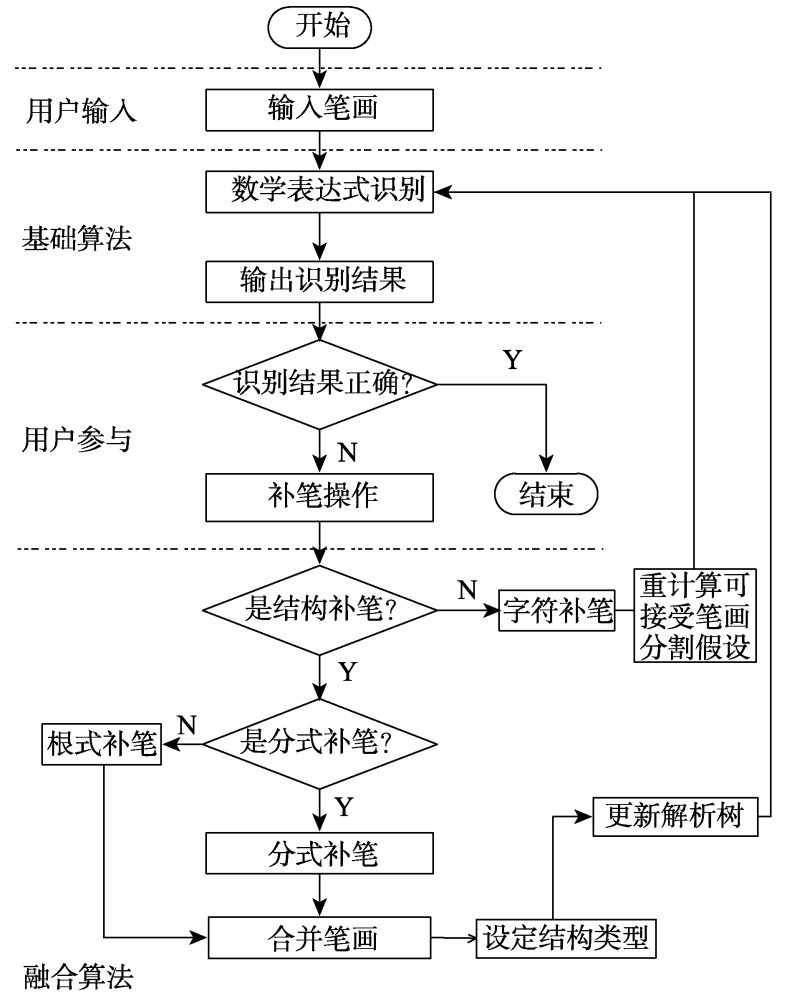

图 5 基于上下文信息的融合方法流程

经过补笔操作后, 手写数学表达式笔画分为 识别正确的笔画 $O_{\mathrm{T}}$ 、错误识别笔画 $O_{\mathrm{F}}$ 和补笔笔画 $o_{\mathrm{N}}$. 在融合算法中, 引人了相应假设.
(1) 与补笔笔画间距离超过最大距离阈值外 的笔画, 被认定为已正确识别, 表示为

$$
\begin{aligned}
& p\left(t^{\prime} \mid O_{\mathrm{T}}\right)=1, O_{\mathrm{T}}=\left\{o_{i} \mid o_{i} \in O, o_{i} \notin O_{\mathrm{F}}\right\}, \\
& O_{\mathrm{F}}=\left\{o_{i} \mid o_{i} \in O, d\left(o_{i}, o_{\mathrm{N}}\right)>L\right\} .
\end{aligned}
$$

其中， $t^{\prime}$ 表示已经识别正确的子表达式解析树.

（2）结合上下文信息，被认定为分式和根式补 笔的补笔笔画，除分式和根式外，其结构内的字符 已被识别正确，其表示为

$$
p(S \mid O)=1, O \in\left\{o_{i} \mid o_{i} \neq o_{\mathrm{r}}, o_{i} \neq o_{\mathrm{f}}\right\} .
$$

其中， $o_{\mathrm{f}}$ 表示已识别分式的笔画; $o_{\mathrm{r}}$ 表示已识别 根式的笔画.

（3）补笔操作过程中, 除了能够改变分式和根 式结构外 (与之对应的算法内结构为上下 below, 内部 inside 和根式 mroot), 其余结构不发生改变.

由于补笔笔画改变了已有算法的部分可接受 笔画分割集合和非终结符编码区域的空间关系, 因此，根据补笔时的相应假设和上下文语义实现 算法的融合.

定义 1. 补笔笔画的区域表示为 $R_{\mathrm{N}}$, 补笔笔 画宽度为 $W$, 错误识别笔画的区域为 $R_{\mathrm{F}}$, 标准化 字符的宽度和高度分别为 $W_{0}$ 和 $H_{0}$.

定义 2. 错误识别笔画 $o_{i}$ 和补笔笔画 $o_{\mathrm{N}}$ 存在 重叠笔画点, 则认为它们是可合并笔画.

定义 3. 如果 $R_{\mathrm{F}} \subseteq R_{\mathrm{N}+\mathrm{F}}, W_{0} \leqslant W, W_{\mathrm{F}} \leqslant W$, 表 示该补笔操作为结构补笔. 其中, $R_{\mathrm{N}+\mathrm{F}}$ 表示补笔 笔画和错误笔画的合并区域; $W_{\mathrm{F}}$ 为错误笔画区域 的宽度.

需要判定模型以区别补笔操作的性质, 这通 常是基于上下文中的语义信息来实现的. 本文根 据补笔笔画与识别错误笔画间的距离、是否存在重 叠以及覆盖区域的包含关系判定补笔操作的性质. 如果补笔操作为结构补笔, 则与错误识别笔画中 表示结构的笔画合并，并修正空间关系概率和解 析树概率，其表示为

$$
\begin{gathered}
o_{\mathrm{c}}=\left\{p_{i} \mid p_{i} \in\left\{o_{\mathrm{st}} \cup o_{\mathrm{n}}-o_{\mathrm{st}} \cap o_{\mathrm{n}}\right\}, o_{\mathrm{st}} \subseteq O_{\mathrm{F}}\right\} ; \\
\left\{\begin{array}{c}
p(r=\text { 'inside', } r=\text { 'mroot' } \mid R T)=1, S_{o_{\mathrm{st}}}=\text { 'lsqrt' } \\
\left\{\begin{array}{c}
p(r=\text { 'below? } \mid M F)=1 \\
p(r=\text { 'mroot' } \mid F Z)=1
\end{array}, \quad S_{o_{\mathrm{st}}}=\right.\text { '|frac' }
\end{array}\right.
\end{gathered}
$$

其中, $p_{i}$ 为笔画中的点; $o_{\mathrm{c}}$ 为结合结构笔画和补 笔笔画的合并笔画; $o_{\text {st }}$ 为错误识别笔画中表示结 构的笔画; $S_{o_{\mathrm{st}}}$ 为错误识别笔画中结构笔画识别的 字符; 'Isqrt' 和 'frac' 是 LATEX 根式和分式字符的 表示; $R, T, M, F$ 和 $Z$ 分别表示根式、根式内容、 
分母、分号和分子所对应的非终结符. 由于补笔后 其分式和根式空间关系已知, 所以错误笔画构造 解析树概率计算方式与基础方法的有所不同.

分式补笔解析楖概率计算公式为

$$
\begin{gathered}
p(t, s)=p\left(M F \mid A_{f}^{\prime}\right) p\left(F Z \mid A_{f}^{\prime \prime}\right) p(r \mid M F) p(r \mid F Z) \times \\
\prod_{(A \rightarrow s, t)} p(\alpha \mid A) \prod_{(A \rightarrow B C, t)} \max _{r} p(B C \mid A) p(r \mid B C) .
\end{gathered}
$$

其中, $p(r \mid M F) p(r \mid F Z)=1$; 非终结符 $A_{f}^{\prime}$ 和 $A_{f}^{\prime \prime}$ 分 别根据分母和分号, 以及分子和分号所表示的非 终结符推导而成.

根式补笔解析楖概率计算公式为

$$
\begin{gathered}
p(t, s)=p\left(R T \mid A_{r}^{\prime}\right) p(r \mid R T) \prod_{(A \rightarrow s, t)} p(\alpha \mid A) \times \\
\prod_{(A \rightarrow B C, t)} \max _{r} p(B C \mid A) p(r \mid B C) .
\end{gathered}
$$

其中, $p(r \mid R T)=1 ; A, B, C$ 为根式内容、分母、分 子内包含的非终结符; $\alpha$ 为终结符; $p(r \mid B C)$ 表 示非终结符 $B$ 和 $C$ 编码区域的空间关系概率; $A_{r}^{\prime}$ 表示根据根式内容和根号所表示的非终结符推导 而成的非终结符.

如果补笔操作是针对错误字符的, 则合并补笔 笔画和错误笔画为集合 $O^{\prime}$ ，重新计算错误识别笔 画的可分割集合 $b_{E}$, 并反馈于基础识别算法, 根据 可分割集合 $b_{E}$ 进行字符识别和表达式识别. 其中,

$$
\begin{gathered}
O^{\prime}=O_{F}+o_{N} ; \\
b_{E}=\left\{b_{i}\left|o_{i}, o_{j} \in O^{\prime}, d\left(o_{i}, o_{j}\right)<L,\right| b_{i} \mid \leqslant L_{\max }\right\} .
\end{gathered}
$$

根据数学表达式上下文语义和用户操作推断 用户补笔的意图, 并结合人的参与和基础方法来 纠正识别错误的数学表达式, 是本文的亮点和核 心. 为了验证本文方法的有效性和可行性, 实验对 本文方法和基础方法在结构识别率和表达式识别 率方面进行了对比分析.

\section{3 用户研究}

为了验证本文方法能够达到预期目标，通过 一项用户研究评估被试在本文方法和基础方法之 间的用户体验、满意度和使用难易程度等. 在基础 方法条件下，被试仅要求书写数学公式，但不能通 过算法识别反馈结果来纠正错误. 而本文方法不 仅有基础方法的用户操作，还包括基于识别结果， 被试使用提出的交互手段参与到识别算法当中去, 特别是公式结构的识别. 此外, 为了确保用户界面 之间的公平比较, 实验围绕同一底层数学公式识
别 Seshat 算法开发了一个手写公式识别交互界面.

主要的研究问题是：(1) 基于本文方法, 用户 能否更容易地参与到识别算法当中去, 即用户认 为交互操作的难易程度? (2) 与基础方法比较, 本 文方法是否表现更好? (3) 本文方法能否满足用户 对手写公式识别的部分需求?

\section{1 研究设计}

本文基于人在回路的手写数学公式识别方法 进行了用户研究, 研究的自变量为数学公式识别 过程中人的交互行为, 主要为删除、撤销、字符补 笔、结构补笔和清空，而因变量为交互时间、准确 度和后问卷调查. 在实验开始前, 参与者需要填写 一份简短的预调查问卷, 以便收集人口统计信息 和是否有笔或笔式界面相关的使用经验.

随后，每个参与者使用手写公式识别交互界 面完成不同方法下的相关任务. 在正式实验前，进 行 5 10 min 的预实验, 主要目的是让参与者熟悉 用户界面和交互技术，以便完成后续的评估任务. 主要任务参与过程分为 2 个阶段: 第 1 阶段是仅依 赖基础方法识别书写的数学公式, 用户在识别完 成后不做任何修改; 第 2 阶段为数学公式识别后, 用户根据识别的反馈信息, 使用本文方法参与到 识别过程中.

在实验结束后, 结合已有评估方法 ${ }^{[2,10,34]}$ 和本 文研究的主要问题, 要求参与者填写一份调查问 卷. 参与者必须回答 4 个方面的问题, 并建议提供 关于任务和识别方法相关的任何主观反馈.

(1) 以 5 分制(1 分表示最困难，5 分表示最简 单)分别评估 5 种交互手段的操作难易程度.

(2) 以 5 分制(1 分表示完全不认可, 5 分表示完 全认可)或“能/不能”选择分别评估识别方法和用户 影响相关的问题.

(3) 以 5 分制(1 分表示完全不认可, 5 分表示完 全认可)实现用户对交互性能的主观评价.

(4) 以 5 分制(1 分表示更偏向于 $C$ 方式，即基 于编辑软件的公式编辑方式; 5 分表示更偏向于 $D$ 方式, 即基于手写识别的公式编辑方法)实现对公 式编辑方式的主观评价.

用户研究更多的是为了衡量本文方法的可行 性和合理性, 而不是衡量 2 种方法的优劣. 因为基 础方法是本文方法的保障，而本文方法是基础方 法的延伸和拓展，2 种方法从不同的角度对同一问 题提出了相应的解决方案. 同时, 本文对用户的意 见和建议进行了汇总，纳人实验报告. 


\section{2 实验设备和用户界面}

(1) 实验设备. 手写数学公式识别评估是在 Windows Surface Pro6 平板电脑上进行的, Intel ${ }^{\circledR}$ CoreTM i5-8250U CPU (1.6 GHz), 8 GB 内存, PixelSense 触控显示屏且配手写笔, 运行 Windows 10 操作系统. 平板电脑放置在一个合适的位置, 可以 使参与者舒适自然地在设备上书写数学公式.

(2) 用户界面. 如图 6 所示, 开发的手写公式 识别交互界面包含手写输人区、识别结果显示区和 交互工具区 3 个模块. 其中, 手写输人区主要用于 参与者输人数学公式(图 6 左下角); 识别结果显示 区主要功能为显示数学公式识别结果, 显示结果 为基于 LATEX 数学描述语言渲染的标准化排版数 学公式(图 6 上部分); 交互工具区主要提供本文设 计的交互工具，方便用户使用并参与到公式识别 当中去(图 6 右下角), 分别为删除、撤销、清空和 帮助.

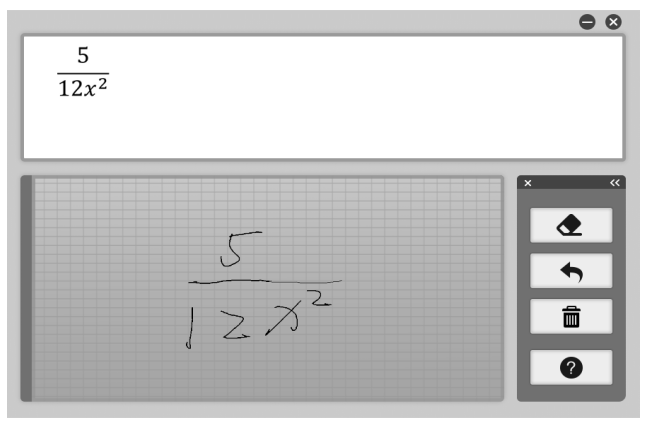

图 6 手写公式识别交互界面

\section{3 参与者}

56 名被试(34 名男性, 22 名女性)和 2 名主试同 意并参与了实验，年龄为 22 48 岁 (平均值 $M=31.30$, 标准差 $\mathrm{SD}=8.84)$. 这些被试来自本技术研发团队 以外的人员，招募于当地大学. 除了参与本次实验 外，被试与本技术研发团队没有其他社会关系。在 研究开始之前, 对被试进行了背景调查. 总体而 言， $69.64 \%$ 的被试对笔设备和手写板比较熟悉， $78.57 \%$ 的被试接受过或正在接受本科以上普通高 等教育(特别是 $50 \%$ 的被试正在接受或接受过硕士 研究生培养教育), 主要从事软件开发、用户界面 设计、人工智能、教育和编辑等方面的工作和学习.

\section{4 任 务}

为了评估本文方法，设计相关的实验任务，主 要分为主试任务和被试任务. 主试任务包括在预 实验阶段指导被试使用设备和交互技术，以及对 被试进行问卷调查. 被试任务主要分为 3 个阶段, 分别为预实验阶段、实验阶段和后问卷调查阶段.
被试在预实验阶段的主要任务是根据主试的指导 熟悉手写设备和交互技术的使用.

实验阶段主要包含 2 个主要任务. (1) 仅依赖 基础方法识别被试书写的数学公式, 如表 1 所示. 在该任务中, 被试只需要根据自己的书写习惯完 成公式的输人即可. (2) 根据基础方法对手写数学 公式的识别结果，被试自由地选择本文提出的交 互技术主动参与到数学公式识别当中去. 被试在 该任务中除了以正常习惯书写数学公式外, 还需 要借助本文提出的交互技术参与到公式识别的环 路中. 需要特别说明的是, 用户在该阶段的 2 个任 务中, 不受类似于文献[10]中因需保证高识别率而 设计的书写规范的约束.

表 1 评估使用的公式

\begin{tabular}{cc}
\hline 标号 & 公式 \\
\hline 1 & $\frac{3}{x+y}$ \\
2 & $\frac{2}{n(n+1)}$ \\
3 & $y=a_{1}^{2} x+a^{2}$ \\
4 & $(a+b)^{2}=a^{2}+2 a b+b^{2}$ \\
5 & $\frac{a_{1}+a_{2}+a^{3}}{1-x}$ \\
6 & $\frac{1}{2(x+3)}+\frac{2}{(x+3)^{2}}$ \\
7 & $\sqrt{\frac{y+z+x^{2}}{x}}$ \\
8 & $\sqrt{\frac{1-\sqrt{1-z}}{z}}$ \\
9 & $\frac{1}{\beta}\left(1+\mathrm{e}^{\frac{-(x-\mu)}{\beta}}\right)$ \\
\hline
\end{tabular}

后问卷调查阶段，根据被试在实验环节的操 作和实验结果, 被试被要求完成关于交互技术和 识别算法等方面的问卷, 并对被试进行了更深人 的后续访谈，主要包括手写公式应用场景、基于手 写公式的交互技术等.

\section{4 实验评估}

\section{1 数 据}

56 名被试参与了手写公式识别实验. 收集的数 据包括被试的基本信息、公式信息和问卷调查表信 息，其中公式信息包含笔画信息和每个公式在不同 算法下的完成时间(time to completion, TTC ${ }^{[10]}$, 完 成时间不包含书写公式的时间. 为了排除被试对 
手写设备的熟悉程度和公式书写速度等方面的差 异性，本文假定每个被试在 2 个算法识别过程中的 公式书写时间相同.

实验对手写公式识别进行了定量化评估和定 性化评估. 定量化评估主要从算法 TTC、结构识别 率(rate of structural analysis, RSA) ${ }^{[25]}$ 和公式识别率 (expression recognition rates, ExpRate) ${ }^{[7]} 3$ 个方面进 行评估，而定性化评估主要通过分析问卷调查表 来评估用户对算法的满意度，对评分数据进行正 态分布检验，结果表明其分布服从正态分布.

\section{2 定量结果}

计算精度有多种方法 ${ }^{[3]}$. 由于本文关心如何以 快速高效的方式提高手写数学公式结构的识别率, 特别是根式和分式结构. 因此, 使用 TTC, RSA, 和 ExpRate 衡量本文方法.

基础方法和本文算法的 RSA 和 ExpRate 如表 2 所示. 本文方法在 RSA 和 ExpRate 上均高于基础 方法, 识别结果在 $95 \%$ 置信度下没有发现显著性 差异(RSA: $t=-1.739, p=0.179$; ExpRate: $t=-2.096$, $p=0.285$ ). 相对于本文使用的基础方法，人在回路 的方法在 RSA 和 ExpRate 上分别提高了 9.26\%和 $13.99 \%$, 对于包含复杂结构的公式，特别是表 2 中 包含分式和根式的公式 6 10, 在 RSA 和 ExpRate 上分别提高了 $15.23 \%$ 和 $21.08 \%$ (RSA: $t=-1.970$, $p<0.05$; ExpRate: $t=-1.996, p<0.05$ ). 该结果说明人 参与到手写数学公式识别当中是可行的, 且能够 提高结构和表达式的识别率. 需要特别说明的是, 对于笔画总数多且包含多个嵌套结构的数学公式, 本文方法要远远优于仅依赖识别功能的方法, 这 是因为人对结构的操作能够降低结构识别的不确 定性. 但是针对“ $z$ ”和“ 2 ” “ $\mu$ ”和“ $u$ ”等相似字符，本 文方法的识别准确率仍然不高, 可以在以后的工 作中结合界面编辑方式进行改进.

表 22 种方法对每个公式的结构和表达式平均识别率

\begin{tabular}{|c|c|c|c|c|}
\hline \multirow{2}{*}{ 公式 } & \multicolumn{2}{|c|}{ 基础 ${ }^{[30]}$} & \multicolumn{2}{|c|}{ 本文 } \\
\hline & RSA & ExpRate & RSA & ExpRate \\
\hline 1 & 100.00 & 94.30 & 100.00 & 99.20 \\
\hline 2 & 95.23 & 91.20 & 100.00 & 98.60 \\
\hline 3 & 95.12 & 90.50 & 100.00 & 98.10 \\
\hline 4 & 88.60 & 82.30 & 93.40 & 91.70 \\
\hline 5 & 89.20 & 84.50 & 93.60 & 92.40 \\
\hline 6 & 82.50 & 78.40 & 92.50 & 92.80 \\
\hline 7 & 83.10 & 75.80 & 92.70 & 90.20 \\
\hline 8 & 81.60 & 77.90 & 90.40 & 91.40 \\
\hline 9 & 75.60 & 60.80 & 86.10 & 83.50 \\
\hline 10 & 50.20 & 40.70 & 78.30 & 64.80 \\
\hline
\end{tabular}

2 种方法在手写公式识别中的平均 TTC 如图 7 所示. 基础方法的 TTC 只包含对手写公式的识别 时间，而本文方法的 TTC 包括依据识别反馈结果 的交互时间和识别时间. 显而易见，对于笔画和结 构越复杂的手写公式, 识别时间越长, 同时人参与 其中的交互时间也越长. 对比 2 种方法, 本文方法 在对包含复杂结构的公式的 TTC 大于基础方法的 $\mathrm{TTC}$, 是因为提出的人在回路方法需要根据识别 结果修正公式结构. 总体而言, 2 种方法在平均 TTC 上存在明显差异 $(t=-1.368, p=0.018)$, 观察平 均 $\mathrm{TTC}$, 前 3 个公式的平均 $\mathrm{TTC}$ 基本保持一致 $(t=-0.076, p=0.905)$, 主要原因在于它们包含简单 的结构和少量的笔画数, 基础方法的识别准确率 比较高, 出现识别错误的概率较小. 2 种方法识别 表 2 中公式 4 10 的平均 TTC 存在显著性差异 $(t=-1.847, p=0.044)$, 存在这种现象的原因在于这 7 个公式包含复杂结构且包含笔画数量较多, 基础 方法在识别中根据距离和可见性划分可允许分割 集合后字符识别阶段对每个可允许分割集合进行 识别, 从而造成基础方法识别时间较长且识别率 较低, 特别是公式 10 中“ $\mu$ ”的识别. 而本文方法需 要在原有识别结果的基础上通过交互技术纠正错 误, 从而会花费较多的时间. 值得说明的是, 本文 方法 TTC 中交互时间占比不足总时间的 36.2\%，每 个公式的平均交互时间不超过 $5 \mathrm{~s}$. 后续工作中会 根据用户对交互时间的满意程度进行定性化评估.

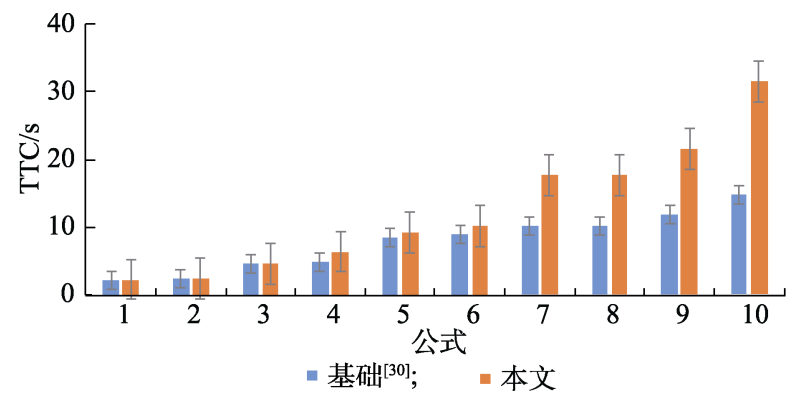

图 72 种方法手写公式识别平均完成时间对比

\section{3 定性结果}

除了 TTC, RSA 和 ExpRate 外, 本文还要求每 位被试根据自己对本文方法的使用情况完成后问 卷调查, 问卷从交互操作难易程度、算法及用户影 响评估和交互性能评估 3 个方面展开。

\subsection{1 交互操作难易程度}

根据问卷调查结果显示，5种交互操作的难易 程度评分最高为 4.32 (评分为 $1 \sim 5$ 分, 得分越高表 示操作越简单). 在 5 种方式中, 撤销和清空仅依 
靠单击按钮完成相关任务, 难易程度平均评分为 3.8 以上, 交互操作难易程度评分如表 3 所示. 因 此，以平均得分 2.5 分为评估基线，用户认为其余 3 种交互操作非常简单，能够使他们容易地参与到 公式识别当中去.

\section{表 3 交互操作难易程度评分(总体)}

\begin{tabular}{lcc}
\hline 交互操作 & 得分平均值 & 标准差 \\
\hline 删除 & 3.64 & 0.97 \\
撤销 & 3.82 & 1.17 \\
字符补笔 & 3.88 & 1.00 \\
结构补笔 & 3.73 & 1.10 \\
清空 & 4.32 & 1.04 \\
\hline
\end{tabular}

为了判断本文方法的优劣性是否与用户对笔 设备或笔式界面的使用经验存在关联，实验根据 “被试是否存在笔设备相关的使用经验”问卷结果 进行分组检查其中相关性，根据曾经是否使用过 笔设备, 将参与者分为参与者 $A$ 和参与者 $B$, 其中, 参与者 $A$ 表示未使用过, 参与者 $B$ 表示使用过. 实 验结果没有发现参与者 $A$ 与参与者 $B$ 之间的评分 存在显著性差异 $(p>0.05)$, 如图 8 所示. 这说明本 文方法与是否有笔式设备或界面方面的经验无关. 但是，不同教育程度的被试对于撤销、分式补笔和 根式补笔 3 种操作的主观评价存在显著性差异 $(p<0.05)$. 本文方法对于低教育程度人群不太友好, 主要原因在于对根式和分式补笔的认知存在一定 的差异性，部分被试在后采访中表示他们不太了 解数学公式的结构分类，以及在生活中很少用到 复杂的数学公式. 对于撤销操作, 参与被试表示它 和删除存在一定的相似性，会与删除操作混淆.

\subsection{2 算法及用户影响评估}

从 10 个方面对算法及用户影响进行了主观性

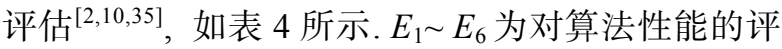
估, $E_{7} \sim E_{10}$ 为算法对用户的影响评估.

对于 $E_{4} \sim E_{6}, 92 \%$ 的被试认为，本文方法能够 满足自己在手写公式识别中的部分需求和提高公

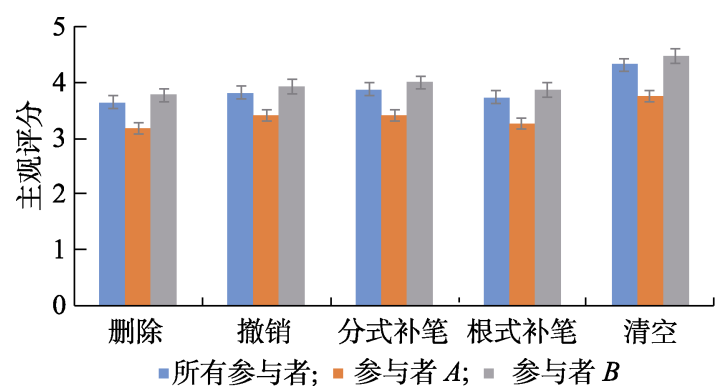

图 8 参与者 $A$ 和 $B$ 对交互操作难易程度的评分结果
表 4 算法及用户影响评估

\begin{tabular}{cl}
\hline 评估项 & \multicolumn{1}{c}{ 主观性评估描述 } \\
\hline$E_{1}$ & 您觉得仅依赖识别的数学公式识别算法, 能满足您 \\
& 对手写数学公式方面的需求 \\
$E_{2}$ & 您觉得人参与到手写公式识别过程中是可行的 \\
$E_{3}$ & 您觉得本文方法比基础方法更容易识别手写数学公式 \\
$E_{4}$ & 您觉得本文方法能否满足自己的需求(输人、识别) \\
$E_{5}$ & 您觉得本文方法能否提高识别正确率 \\
$E_{6}$ & 您觉得本文方法能否有助于您理解所涉及的操作 ${ }^{[35]}$ \\
$E_{7}$ & 您觉得本文方法没有增加您的认知负荷 \\
& 您觉得本文方法不会引入不必要的麻烦(交互困难, \\
$E_{8}$ & 打断思考) \\
$E_{9}$ & 您觉得本文方法很自然(删除、重写、补笔等) \\
$E_{10}$ & 您觉得本文方法符合您的书写习惯 \\
\hline
\end{tabular}

式的识别率, 且能够帮助他们理解分式和根式结 构的补笔操作. 值得一提的是, $E_{1}$ 的平均评分为 2.98( $\mathrm{SD}=1.30)$, 与文献[3]调研结果存在一些差异, 具体为用户觉得现有识别率不高的方法也能够在 一定程度上满足自己的需求. 存在差异的原因可 能与被试在后续参与到数学公式识别过程中有关, 认为低的初始识别率可以通过自己的参与来提高; 以及用户仅需要对简单公式进行识别, 而现有公 式识别方法能够胜任这些需求. 对于 $E_{2}$ 和 $E_{3}$, 被 试认为人参与其中的方法是可行的, 且参与其中 的方法优于基础方法, 平均评分分别为 $3.84(\mathrm{SD}=$ 1.172)和 3.79( $\mathrm{SD}=1.107)$. 而对于本文方法增加了 认知负荷 $\left(E_{7}\right)$ 和引人了不必要的麻烦 $\left(E_{8}\right)$, 评分高 于 2.50 , 分别为 $3.07(\mathrm{SD}=1.32)$ 和 $3.25(\mathrm{SD}=1.24)$; 对于 $E_{9}$ 和 $E_{10}$, 平均评分接近 4 分. 根据 $E_{7} \sim E_{10}$ 的 调查结果，本文方法符合用户书写习惯，且没有增 加过多的认知负荷.

除了 $E_{8}$ 外，没有发现教育程度和笔使用经验 对评估结果存在显著性差异. 教育程度在 $E_{8}$ 评估 结果中存在显著性差异 $(t=2.606, p=0.035)$, 其原因 可能是不同教育程度的被试在生活工作中需要解 决的问题不同，例如，被试建议“手写公式识别系 统可增加公式编辑后的图片和 LATEX 等格式的结 果输出”和“希望增加复杂公式推导功能”等.

\subsection{3 交互性能评估}

如表 5 所示，实验从交互设计合理性、交互时 间满意度、非结构补笔的满意度、结构补笔的难易 度和整体交互的难易度 5 个方面进行了交互性能 评估，总体评分在 4 分左右.

分析结果表明, 本文在手写数学公式识别过 程中引人人参与的方式设计合理，操作简单，能够 在很少的交互时间内提高结构和公式的识别率, 
表 5 交互性能评估(总体)

\begin{tabular}{lcc}
\hline \multicolumn{1}{c}{ 交互性能 } & 得分平均值 & 标准差 \\
\hline 交互设计的合理性 & 4.04 & 0.972 \\
交互时间的满意度 & 3.70 & 1.127 \\
非结构补笔的满意度 & 4.07 & 0.806 \\
结构补笔的满意度 & 3.93 & 0.988 \\
整体交互的满意度 & 4.07 & 0.931 \\
\hline
\end{tabular}

除了结构补笔的满意程度低于 4 分以外, 其余均高 于 4 分, 可能原因在于相对于其他操作, 结构补笔 的操作相对较难. 同时, 本文方法能够满足不同教 育程度人群对手写数学公式的识别需求, 且与是 否有手写设备操作经验没有关联. 例如, 结构补笔 的满意程度在不同教育程度的被试评估结果中没 有发现显著性差异 $(t=-0.703, p=0.874)$.

\subsection{4 公式编辑方式对比}

为了比较公式编辑方式的易用性、喜爱程度和 使用效率等, 本文还研究了基于手写识别的公式 编辑方式与基于公式编辑软件的编辑方式的主观 问卷调查. 因此，实验从用户喜爱程度、易用性、 使用自然度、使用效率、输人速度、认知负担等方 面进行了用户主观评价 ${ }^{[36]}$, 如表 6 所示, 1 分表示 更偏向于 $C$ 方式, 即基于编辑软件的公式编辑方 式(MathType, Office 等), 5 分表示更偏向于 $D$ 方式, 即基于手写识别的公式编辑方法.

表 6 公式编辑方式的主观评估

\begin{tabular}{lcc}
\hline \multicolumn{1}{c}{ 主观评估 } & $\begin{array}{c}\text { 得分 } \\
\text { 平均值 }\end{array}$ & $\begin{array}{c}\text { 标准 } \\
\text { 差 }\end{array}$ \\
\hline 您更喜欢的公式编辑方式 & 3.68 & 1.42 \\
您觉得更容易使用的公式编辑方式 & 3.64 & 1.28 \\
您觉得使用效率更高的公式编辑方式 & 3.53 & 1.32 \\
您觉得输人速度更快的公式编辑方式 & 3.70 & 1.43 \\
您觉得输人更自然的公式编辑方式 & 3.86 & 1.24 \\
您觉得输人更轻松的公式编辑方式 & 3.79 & 1.22 \\
您觉得降低了您的认知负荷的公式编辑方式 & 3.55 & 1.34 \\
您觉得更加符合习惯的公式编辑方式 & 3.61 & 1.33 \\
\hline
\end{tabular}

实验从 8 个方面进行了用户主观性评估，总体 而言，参与评估的用户更偏向于基于手写识别的 公式编辑方式, 认为这种编辑方式在输人速度、使 用轻松程度、易用性等多个方面优于基于编辑软件 的公式编辑方式 $(M>3.5)$, 同时, 问卷调查结果与 笔输人方式的评估结果 ${ }^{[37-38]}$ 基本保持一致. 根据 对“是否有手写设备操作经验”和“不同教育程度人 群”的主观评价进行差异性分析发现，除了不同学 历人群对 $C$ 方式和 $D$ 方式的偏爱程度存在差异外 $(t=0.822, p=0.035$; 高水平教育程度人群 $M=3.61$,
$\mathrm{SD}=1.530$; 低水平教育程度人群 $M=3.92, \mathrm{SD}=0.996$ ), 在其他方面没有发现显著性差异. 对于不同教育 程度的人群, 高水平教育程度人群在喜爱程度上 得分较低, 可能原因在于使用 Office 或 MathType 等软件的频率更高, 相对于基于公式识别的编辑 方式, 使用编辑软件的准确率完全可控, 且与用户 的编辑内容相关. 而对于低教育水平的用户，基于 公式识别的编辑方式比非笔输人编辑软件更容易 操控, 且不需要频繁地查找公式复杂结构的模板, 所以更喜欢使用笔输人数学公式.

\section{5 讨 论}

本文方法为了解决仅仅依靠识别算法不能满 足用户对手写数学公式识别需求的问题, 在识别 过程中引人人的参与, 特别是在公式结构识别方 面. 基于被试反馈的一致结论是, 本文方法能够满 足用户的部分需求，引人人参与的方式设计合理， 操作简单, 且能够在很短的时间内参与其中. 从定 量化评估结果来看, 结构识别率的提升对公式整 体识别率的提高有不可估量的帮助作用，在仅依 靠识别算法不能完美解决结构识别时, 引人人的 参与或许是一种解决的途径. 同时, 调查问卷结果 表明，操作简单、交互时间短、不增加用户认知负 荷且能够解决特定问题的交互方式是用户参与到 机器不能完美解决的任务的重要手段, 如人在手 写公式识别任务中的参与.

在交互操作难易程度评估中，被试认为删除 操作很难使用, 如“擦除不好用, 特别是对细微部 分的擦除” “删除操作对于细微且很靠近的笔画难 以处理”和“擦除的精细化操作，如何更人性化”等 反馈, 主要是因为设计的删除操作不只针对单个 笔画, 还可以删除笔画中的部分点. 后续设计中可 以根据删除内容进行细分删除操作, 如删除笔画 块、删除单笔和删除局部点等. 针对撤销和清空操 作, 本文采用按钮方式触发, 主要是因为撤销和清 空操作属于一键式(one touch)任务, 在使用按钮的 情况下可能表现更佳. 如果使用笔手势操作, 还需 要面对笔手势识别不准确的问题. 对于是否使用 笔手势代替按钮, 以及如何结合按钮和笔手势在 相关任务中实现更好的协作, 需要在后续的研究 中验证.

对收集的 560 个公式进行了分析(本文用于实 验和收集的数据远远超过文献 [10]中包含的数量, 该文献包含 10 个数学公式, 仅有 12 位被试). 尽管 
得到较好的实验结果，但是并不能囊括所有公式， 特别是矩阵操作、三角函数等. 文献[14]表明，在 数学公式识别中最常见的错误是结构上的混淆, 而在符号识别中最常见的是大小写错误和相似外 观的错误 ${ }^{[13]}$. 本文主要针对这 2 点提出了人在回 路的在线手写公式识别方法, 通过引人人的参与 解决机器容易混淆的错误. 因此, 在数学公式识别 任务中选择的公式主要为存在结构和相似字符的 公式，如分式结构、根式结构、包含“ “ $a$ ”和“ $\mu$ ”的公 式等. 另外, 像绝对值、三角函数和求和公式等, 主要区别在于公式中包含的字符不同，而不是包 含了特殊的结构. 需要说明的是, 本文更多地关注 人在回路方法是否能够提高公式识别的准确率和 满足用户的需求，实验选取公式时忽略了三角函 数、绝对值等函数, 将给本文带来了一定限制. 可以 在以后的工作中结合需要解决的问题和公式类型, 尽可能全面地选择任务中涉及的公式和数据.

对于引人人参与的手写数学公式识别方法, 选用 Seshat 方法的原因在于, Seshat 是一个基于符 号分割-符号识别-结构解析 3 阶段的方法, 该方法 可以根据用户参与的意图和上下文理解，在不同 阶段引人人的参与提高手写数学公式识别的准确 率. 但是对于端到端的方法 ${ }^{[7,15,39]}$, 很难根据用户 意图调整或修改模型的结构. 对于将人在回路的 思想迁移到其他识别方法的有效性和合理性，可 以从 2 个方面来说明: (1) 识别方法不能完全处理 用户在书写过程中的随机错误，并且仅有用户知 道自己想要表达的内容; (2) 用户参与到识别当中, 可以为识别方法提供有用的反馈信息, 如补笔操 作后为方法提供了明确的结构信息等，而这些反 馈信息能够在不同阶段为方法提供帮助.

根据相关研究, 数学公式识别研究主要集中 于仅依赖机器的识别算法和用户输人约束 2 方面, 并没有在数学公式识别过程引人人干预的研究. Seshat 方法仅对公式进行识别, 除了用户输人外, 没有其他人介人的方式. 因此，除了与基线方法 Seshat 进行比较外, 实验只能通过用户的主观评价 判断人在回路方法的有效性. 由于在线手写数学 公式识别面临的多种问题, 特别是在数学公式书 写中出现偶然性错误和包含复杂结构的情况下, 关注既定流程规范和加速的现有方法很难满足用 户的需求. 但是，用户能够确定现有识别算法的错 误结果, 并且能够通过交互手段适当地纠正结果, 这也弥补了仅依赖机器识别方法与用户最终需求 间的鸿沟. 需要说明的是, 本文方法中用户的介人
方式仍然是对输人笔画的操作, 很难解决如 “ $\mu$ ”和 “ $u$ ”的识别错误问题，因此，后续的研究可以混合 手写识别方法和界面编辑方式, 结合两者的优势 解决仅依靠修改笔画无法满足用户需求的问题.

被试针对公式识别问题提出了宝贵的建议, 如“希望根式补笔等交互方式更人性化, 加人必要 的错误信息提示” “数学公式的支持范围可分级完 成, 小学到大学的公式的复杂程度和识别率肯定 不同; 限定范围，提高简单公式识别率和交互效 率” “基础方法识别度低, 需要写完整的公式较为 麻烦, 可以尝试加人联想功能, 写出一部分, 即可 提示一些可选项”等. 希望在后续研究中能够进一 步完善人在回路的手写公式识别中遇到的问题.

\section{6 结 语}

数学手写体识别是计算机数学输人的一种主 要方法. 虽然近年来在识别方面取得了长足的进 步，但由于用户书写字符顺序不一致、书写字符风 格迥异、数学公式的二维结构信息复杂和全局信息 缺乏等问题, 数学公式识别仍然处于识别错误很 常见的局面. 因此, 本文通过设计友好的交互方 式, 在手写数学公式识别过程中引人了人的参与, 与机器共同完成识别任务. 实验中, 定量化结果表 明, 人参与的识别方式能够提高结构和表达式识 别率，分别提高了 $9.26 \%$ 和 $13.99 \%$, 对于包含复杂 结构的公式, 特别是包含分式和根式的公式, 在结 构和表达式识别率上分别提高了 $15.23 \%$ 和 $21.08 \%$. 定性化评估结果表明，本文方法能够满 足不同教育程度人群对手写数学公式的部分需求, 引人人参与的方式设计合理, 操作简单, 且能够在 很短的时间内参与其中. 研究结果表明, 人参与到 手写公式识别过程中是可行的. 接下来的工作会 在人参与的手写识别公式上继续研究, 希望通过 引人人的参与弥补方法在公式识别中的不足.

\section{参考文献(References):}

[1] Anderson R H. Syntax-directed recognition of hand-printed two-dimensional mathematics[M] //Interactive Systems for Experimental Applied Mathematics. Amsterdam: Elsevier, 1968: 436-459

[2] Zhelezniakov D, Cherneha A, Zaytsev V, et al. Evaluating new requirements to pen-centric intelligent user interface based on end-to-end mathematical expressions recognition[C] //Proceedings of the 25th International Conference on Intelligent User Interfaces. New York: ACM Press, 2020: 212-220 
[3] Anthony L, Yang J, Koedinger K R. A paradigm for handwriting-based intelligent tutors[J]. International Journal of Human Computer Studies, 2012, 70(11): 866-887

[4] Mahdavi M, Zanibbi R, Mouchere H, et al. ICDAR 2019 CROHME + TFD: competition on recognition of handwritten mathematical expressions and typeset formula detection[C] // Proceedings of the International Conference on Document Analysis and Recognition. Los Alamitos: IEEE Computer Society Press, 2019: 1533-1539

[5] Wang D H, Yin F, Wu J W, et al. ICFHR 2020 competition on offline recognition and spotting of handwritten mathematical expressions - OffRaSHME[C] //Proceedings of the 17th International Conference on Frontiers in Handwriting Recognition. Los Alamitos: IEEE Computer Society Press, 2020: 211-215

[6] Hong Z L, You N, Tan J, et al. Residual BiRNN based Seq2Seq model with transition probability matrix for online handwritten mathematical expression recognition[C] //Proceedings of the International Conference on Document Analysis and Recognition. Los Alamitos: IEEE Computer Society Press, 2019: 635-640

[7] Zhang J S, Du J, Dai L R. Track, attend, and parse (TAP): an end-to-end framework for online handwritten mathematical expression recognition[J]. IEEE Transactions on Multimedia, 2019, 21(1): 221-233

[8] Phan K M, Nguyen C T, Le A D, et al. An incremental recognition method for online handwritten mathematical expressions $[\mathrm{C}] / /$ Proceedings of the 3rd IAPR Asian Conference on Pattern Recognition. Los Alamitos: IEEE Computer Society Press, 2015: 171-175

[9] Le A D, Nguyen H D, Indurkhya B, et al. Stroke order normalization for improving recognition of online handwritten mathematical expressions[J]. International Journal on Document Analysis and Recognition, 2019, 22(1): 29-39

[10] Taranta E M, LaViola J J. Math boxes: a pen-based user interface for writing difficult mathematical expressions[C] //Proceedings of the 20th International Conference on Intelligent User Interface. New York: ACM Press, 2015: 87-96

[11] Bahashwan M, Abu-Bakar S, Sheikh U. Efficient segmentation of Arabic handwritten characters using structural features[J]. International Arab Journal of Information Technology, 2017, 14(6): 870-879

[12] de-Arteaga M, Fogliato R, Chouldechova A. A case for humans-in-the-loop: decisions in the presence of erroneous algorithmic scores[C] //Proceedings of the CHI Conference on Human Factors in Computing Systems. New York: ACM Press, 2020: 1-12

[13] Zhang T, Mouchère H, Viard-Gaudin C. A tree-BLSTM-based recognition system for online handwritten mathematical expressions[J]. Neural Computing and Applications, 2020, 32(9): 4689-4708

[14] Alvaro F, Zanibbi R. A shape-based layout descriptor for classifying spatial relationships in handwritten math[C] // Proceedings of the ACM Symposium on Document Engineering. New York: ACM Press, 2013: 123-126

[15] Alvaro F, Sanchez J A, Benedi J M. Recognition of online handwritten mathematical expressions using 2D stochastic context-free grammars and hidden Markov models[J]. Pattern Recognition Letters, 2014, 35(1): 58-67

[16] Wang J M, Du J, Zhang J S. Stroke constrained attention network for online handwritten mathematical expression recognition[OL]. [2020-11-23]. https://arxiv.org/abs/2002.08670
[17] Naderan E. Online handwritten mathematical expressions recognition system using fuzzy neural network[OL]. [2020-11-23]. https://arxiv.org/abs/1707.03088v2

[18] O'Connell T, Li C J, Miller T S, et al. A usability evaluation of AlgoSketch: a pen-based application for mathematics[C] // Proceedings of the 6th Eurographics Symposium on Sketch-Based Interfaces and Modeling. New Orleans: DBLP, 2009: 149-157

[19] Zeleznik R C, Miller T, Li C J, et al. MathPaper: mathematical sketching with fluid support for interactive computation[C] // Proceedings of the 9th International Symposium on Smart Graphics. Heidelberg: Springer, 2008: 20-32

[20] Klie J C, de Castilho R E, Gurevych I. From zero to hero: human-in-the-loop entity linking in low resource domains[C] // Proceedings of the 58th Annual Meeting of the Association for Computational Linguistics. Stroudsburg: Association for Computational Linguistics, 2020: 6982-6993

[21] Doan A H, Ardalan A, Ballard J, et al. Human-in-the-loop challenges for entity matching: a midterm report[C] //Proceedings of the 2nd Workshop on Human-In-the-Loop Data Analytics. New York: ACM Press, 2017: Article No.6

[22] Lee M H, Siewiorek D P, Smailagic A, et al. Interactive hybrid approach to combine machine and human intelligence for personalized rehabilitation assessment[C]//Proceedings of the ACM Conference on Health, Inference, and Learning. New York: ACM Press, 2020: 160-169

[23] Benedikt L, Joshi C, Nolan L, et al. Human-in-the-loop AI in government: a case study[C] //Proceedings of the 25th International Conference on Intelligent User Interfaces. New York: ACM Press, 2020: 488-497

[24] Mosier K L, Skitka L J, Heers S, et al. Automation bias: decision making and performance in high-tech cockpits[J]. The International Journal of Aviation Psychology, 1998, 8(1): 47-63

[25] Madras D, Pitassi T, Zemel R. Predict responsibly: improving fairness and accuracy by learning to defer[C] //Proceedings of the Advances in Neural Information Processing Systems. Cambridge: MIT Press, 2018: 6147-6157

[26] Xin D, Ma L T, Liu J L, et al. Accelerating human-in-the-loop machine learning: Challenges and opportunities[C]//Proceedings of the 2nd Workshop on Data Management for End-to-End Machine Learning. New York: ACM Press, 2018: Article No.9

[27] Li W C, Sadigh D, Sastry S S, et al. Synthesis for human-in-the-loop control systems[C] //Proceedings of International Conference on Tools and Algorithms for the Construction and Analysis of Systems. Heidelberg: Springer, 2014: 470-484

[28] Toxtli C, Monroy-Hernández A, Cranshaw J. Understanding chatbot-mediated task management[C] //Proceedings of the CHI Conference on Human Factors in Computing Systems. New York: ACM Press, 2018: Article No.58

[29] Gil M, Pelechano V, Fons J, et al. Designing the human in the loop of self-adaptive systems[C] //Proceedings of International Conference on Ubiquitous Computing and Ambient Intelligence. Heidelberg: Springer, 2016: 437-449

[30] Álvaro F, Sánchez J A, Benedí J M. An integrated grammar-based approach for mathematical expression recognition[J]. Pattern Recognition, 2016, 51: 135-147

[31] Álvaro F, Sánchez J A, Benedí J M. Offline features for classifying handwritten math symbols with recurrent neural networks[C] //Proceedings of the 22nd International Conference on Pattern Recognition. New York: ACM Press, 2014: 2944-2949

(下转第 1794 页) 